\title{
A stigmatic ultraviolet-visible monochromator for use with a high brightness laser driven plasma light source
}

\author{
Jun Feng, ${ }^{1}$ J. Nasiatka, ${ }^{1}$ Jared Wong, ${ }^{1}$ Xumin Chen, ${ }^{1}$ Sergio Hidalgo, ${ }^{1}$ T. Vecchione,${ }^{2}$ \\ H. Zhu, ${ }^{3}$ F. Javier Palomares, ${ }^{4}$ and H. A. Padmore ${ }^{1}$ \\ ${ }^{1}$ Lawrence Berkeley National Laboratory, Berkeley, California 94720, USA \\ ${ }^{2}$ SLAC National Accelerator Laboratory, Menlo Park, California 94025, USA \\ ${ }^{3}$ Energetiq Technology, Inc, Woburn, Massachusetts 01801, USA \\ ${ }^{4}$ Instituto de Ciencia de Materiales de Madrid (CSIC), Sor Juana Inés de la Cruz, 3, 28049 Madrid, Spain
}

(Received 10 May 2013; accepted 22 July 2013; published online 16 August 2013)

\begin{abstract}
Laser driven plasma light sources offer highly intense output in the UV-visible region combined with a source size as small as $100 \mu \mathrm{m}$. In order to effectively use the small source size in high brightness applications, a stigmatic monochromator and focusing system must be used. Here we describe a simple brightness preserving optical system that should be useful across a broad range of applications. The output flux of this system is between $6 \times 10^{11} \mathrm{ph} / \mathrm{s}$ and $4 \times 10^{12} \mathrm{ph} / \mathrm{s}$ with a spectra resolution of $1.7 \mathrm{~nm}$ and field spot size of $0.1 \mathrm{~mm}$ from the UV to the VIS spectra range. (C) 2013 AIP Publishing LLC. [http://dx.doi.org/10.1063/1.4817587]
\end{abstract}

\section{INTRODUCTION}

The design of low emittance and high quantum efficiency photocathode is one of the key challenges for next generation accelerator-based light source. ${ }^{1}$ Along with steady improvement of understanding for traditional photocathodes such as cesiated $\mathrm{GaAs}^{2}$ and copper, ${ }^{3}$ new types of photocathode such as plasmon-enhanced photocathode ${ }^{4,5}$ and alkali-antimonides photocathode ${ }^{6,7}$ have been investigated recently. In order to study the emission threshold, quantum efficiency, and emittance of these photocathodes, a light source that provides enough photons in a narrow bandwidth in the deep UV-VIS range and has a small spot size is required.

Traditional light sources in the UV-visible range for applications requiring a broad wavelength tuning range are typically based on arc lamps. The source size of the lamp is primarily related to the electrode size due to the requirements of cooling and lifetime, and this typically results in source sizes of around $1 \mathrm{~mm}$. A recent advance has been the development of the laser driven plasma light source. ${ }^{8}$ In this source, a CW near infrared (NIR) laser of typically $50 \mathrm{~W}$ is focused with a high numerical aperture lens into a fused silica bulb filled with high pressure rare gas. Following the initial ignition of plasma in the bulb by a traditional arc lamp igniter, the highly focused NIR laser beam is absorbed by the plasma and heats the medium to a very high temperature, at which point the initiation arc is terminated and the plasma is self sustaining. Plasma temperatures above $10000 \mathrm{~K}$ can be achieved. Atomic emission spectral structures are Stark broadened into a spectrum that is representative of a low emissivity black body with emission from the VUV to near IR wavelengths. The laser beam is strongly focused so that the ultimate source size is limited only by the radial cooling of the plasma, and typically sizes of around $100 \mu \mathrm{m}$ in FWHM can be achieved. The challenge therefore is to monochromatize this high brightness source and preserve the brightness throughout the whole optical system over a wide wavelength range from the source to a sample. Traditional monochro- mators have been based on spherical optics, for example, in spherical grating systems such as the Seya-Namioka design ${ }^{9}$ or in plane grating single mirror spherical collimating designs such as the Ebert-Fastie design ${ }^{10}$ or double spherical collimating mirror configurations such as the Czerny-Turner. ${ }^{11}$ All of these designs offer good broad band tuning, but suffer from astigmatism to varying degrees. In the present application we chose the Czerny-Turner configuration due to its inherent flexibility. Many designs have been proposed to overcome the inherent astigmatism in the Czerny-Turner design, ${ }^{12-15}$ but either the correction is only complete over a limited wavelength range, or the transmission optics limits the deep UV performance. Toroidal mirrors have been employed to replace the spherical mirrors to eliminate astigmatism in an asymmetric Czerny-Turner spectrometer; ${ }^{15}$ however, such elements tend to be expensive. In contrast to other very simple and cost effective efforts made to reduce significantly astigmatism, i.e., to force the sagittal and tangential foci to coincide, with use of a transmissive cylindrical focusing optic ${ }^{13}$ or by combining divergent illumination ${ }^{14}$ with appropriate detector orientation and location and grating to focusing mirror separation, ${ }^{12}$ we have adopted here an off-axis paraboloid mirror focusing scheme to provide perfect collimation and focusing. The widespread availability of free-form diamond turned metal optics or replicated optics with low figure error makes this a very cost effective solution for a wide wavelength range stigmatic monochromator with applications across a broad range of scientific measurement areas. The advantage over conventional designs is that the system is completely stigmatic over a very wide wavelength range and allows us to preserve the inherent brightness of a new generation of very high brightness sources based on laser plasmas.

\section{SYSTEM DESIGN AND CONSTRUCTION}

Figure 1 shows a schematic of the optical system of the stigmatic Czerny-Turner monochromator. It consists of 
a laser-driven light source (Energetiq EQ-1500), a $150 \mu \mathrm{m}$ diameter entrance pinhole, four paraboloid mirrors with $200 \mathrm{~mm}$ effective focus length, one plane grating with density of $1200 \mathrm{l} / \mathrm{mm}$, and a $100 \mu \mathrm{m}$ diameter exit pinhole or a $100 \mu \mathrm{m}$ core diameter optical fiber. The first pair of $90^{\circ}$ paraboloidal mirrors is used to produce a $1: 1$ focus at the entrance pinhole. The function of this pinhole is to remove the non-Gaussian tails of the source emission, caused by the equilibrium between plasma heating by the NIR drive laser and plasma cooling, as well as diffuse scatter from components within the source. Light through the pinhole is then collimated using another $90^{\circ}$ paraboloid mirror, and the collimated light is directed to a rotatable plane grating which disperses the white spectrum. Light from the grating is refocused by an identical paraboloid mirror and is focused onto an exit aperture. Two types of exit aperture have been used, one in which a slit is used with free-space transmission to relay focusing optics and one in which we couple directly into a $100 \mu \mathrm{m}$ core diameter deep UV-capable fiber. Free space propagation is used when we want to access the deepest UV wavelengths lower than $200 \mathrm{~nm}$, and fiber optic coupling is used for longer wavelength application. The $90^{\circ}$ paraboloidal mirrors have an $\mathrm{MgF}_{2}$ over-coated on $\mathrm{Al}$ substrate to enhance the deep UV reflectivity. The system is designed to have an $\mathrm{f}$ number of approximately f/5.7. The pre-mirrors (mirrors 1 and 2 in Figure 1) are pre-aligned on aluminum support blocks, and the blocks are aligned to each other with gauge blocks. This design assures sufficient parallelism of the two optical surfaces. The collimating and refocusing mirrors (mirrors 3 and mirror 4 in Figure 1) are mounted on a machined block that is used to pre-align each of the two paraboloidal mirrors. This block is aligned with respect to the entrance pinhole and the first two paraboloidal mirrors using normal mechanical metrology techniques. The grating is mounted on a system that allows both in-vacuum motorized rotation for scanning the wavelength and manual adjustment of the grating yaw (rotation about the grating pole). All of these mechanical systems are rigidly bolted to a custom optical table. In order to maximize transmission through the entrance pinhole, the EQ-1500 light source is mounted on an $x-y-z$ stage and the focus size at the pinhole is minimized by positioning of the light source. Due to the small source size, the alignment of the source to the optics is a critical part of the alignment procedure. The grating yaw is adjusted so that the zero order and monochromatic order are in the same plane in the nondispersive direction at the exit aperture. This is a particularly critical alignment due to the use of an exit pinhole defined by the core diameter of fiber with $100 \mu \mathrm{m}$. An incorrect yaw adjustment would cause the monochromatic beam to move off the fiber when the wavelength was scanned. This effect can easily be reduced to a negligible level using the manual yaw adjustment. The aluminum optical baseplate is mounted via a three point kinematic mounting to the stainless steel baseplate of a small vacuum tank. The system is typically evacuated to a pressure of $10^{-5} \mathrm{mBar}$ by a small turbo pump and then sealed off for operation. Light from the plasma source is transmitted into the vacuum system through a sapphire window that gives high transmission down to $150 \mathrm{~nm}$ wavelength. Light from the system is transmitted through a deep UV fiber and

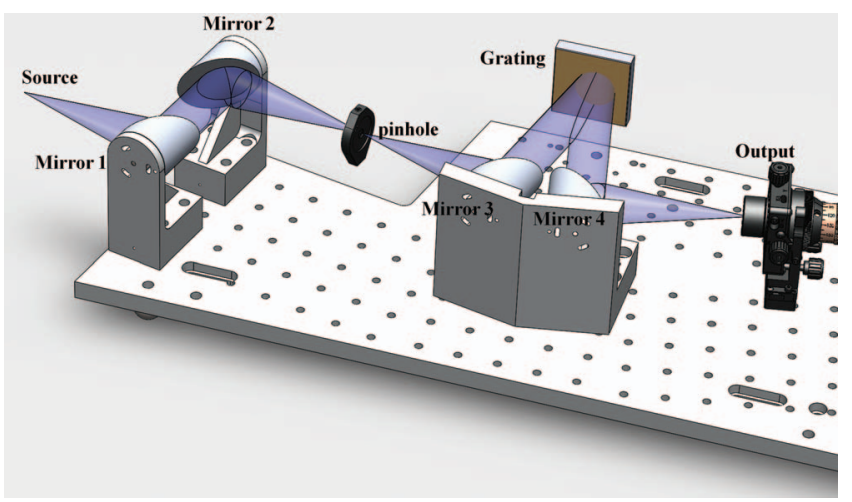

FIG. 1. Optical layout of the high-brightness UV-VIS monochromator system.

coupled out through the wall of the vacuum chamber through a fiber bulkhead connector.

The use of the double paraboloidal pre-mirror system (mirror 1 and 2 in Figure 1) was a pragmatic choice, given by the availability of high quality paraboloidal mirrors. In addition, the system as designed can be used with a $90^{\circ}$ deflecting ellipsoidal relay mirror to increase deep UV throughput and ease alignment. We have also used the system without an entrance pinhole, eliminating mirrors 1 and 2 and effectively placing the plasma source at the focal spot of mirror 3 . This results in a system with higher transmission, but with a somewhat Lorentzian line shape, due to the nature of the light distribution from the plasma source. The light source itself is purged with nitrogen to avoid the production of ozone, and the interspace between the light source and the sapphire window is also purged. For optical alignment at atmospheric pressure, a UV absorbing glass filter is placed at the exit of the plasma source. Figure 2 shows a picture of the overall system and the optical layout inside the system.

The dispersion in this constant included angle geometry system is given by

$$
\frac{d \lambda}{d s}=\frac{g}{m r} \cos \beta, \quad \text { where } \quad \beta=\arcsin \left(\frac{m \lambda}{2 g \cos \theta}\right)-\theta,
$$

where $\mathrm{s}$ is the distance in the dispersive direction at the exit plane, $\mathrm{g}$ is the grating periodicity, $\mathrm{m}$ is the diffraction order, $\mathrm{r}$ is the mirror to exit plane distance, $\beta$ is the angle of diffraction, and $\theta$ is half of the included angle between the incident and diffracted beams. With $\mathrm{g}=833 \mathrm{~nm}(1200 \mathrm{l} / \mathrm{mm})$, $\mathrm{r}=100 \mathrm{~mm}, \mathrm{~m}=1$, and $\theta=3.88^{\circ}$, the reciprocal linear dispersion is $8.3 \mathrm{~nm} / \mathrm{mm}$ and is approximately wavelength independent as $\cos \beta$ tends to 1 . For a fiber size of $0.1 \mathrm{~mm}$ core diameter, the exit slit limited resolution is $0.83 \mathrm{~nm}$. The same equation can be used to assess the entrance slit limited resolution with $\alpha$ substituted for $\beta$, and as the entrance slit to the collimating mirror distance is the same as the focusing mirror to the exit slit distance, the source limited resolution for the $0.15 \mathrm{~mm}$ diameter entrance pinhole size is $1.24 \mathrm{~nm}$. The quadratic sum gives the system resolution as $1.5 \mathrm{~nm}$.

Another aspect that can contribute to the resolution is the geometric aberrations of the system. These double paraboloid mirrors and the plane grating system clearly have no point 

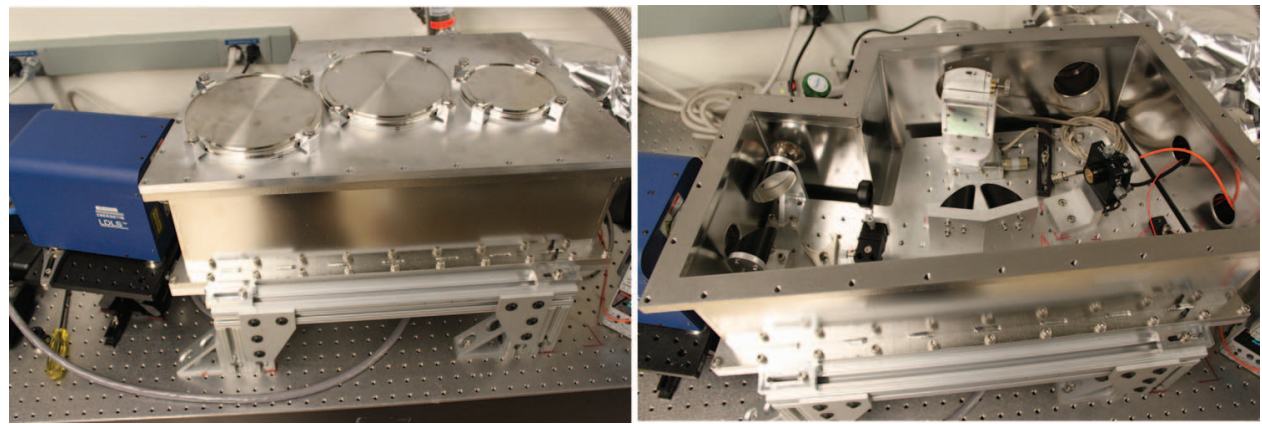

FIG. 2. External picture of the monochromator and source (left) and a close up picture showing the arrangement of the optical elements (right).

aberrations, but it will have aberrations due to the size of the source field. The system was therefore modeled using the raytracing code Shadow. ${ }^{16}$ A square pinhole source is used in the simulation, so that field aberrations at the extreme points could be assessed. The full numerical aperture of $f / 5.7$ was uniformly filled with rays. An example of the performance of the system is shown in Figure 3 for 5 energies around central photon energy of $5.0 \mathrm{eV}$, assuming a $1200 \mathrm{l} / \mathrm{mm}$ grating.

The fiber exit slit is located on-axis at coordinate $(0,0)$, and it can be seen from Figure 3 that a faithful image of the $0.1 \mathrm{~mm}$ diameter entrance slit in the non-dispersive direction is preserved. In the dispersive direction, again, for the onenergy rays at $5 \mathrm{eV}$, the sharp truncation of the central image indicates essentially aberration free imaging. For rays outside the fiber collection aperture, i.e., at 4.8, 4.9, 5.1, and $5.2 \mathrm{eV}$, the image quality becomes progressively worse, in both dispersive and non-dispersive directions. This is of no concern in the present case, as we are only interested in the on-axis use of this system as a monochromator.

\section{RESULTS AND DISCUSSION}

A key element of the present application is that we need maximum throughput especially in the deep UV with preserving the light source brightness. An important aspect therefore is the transmission of the refocused plasma source onto the

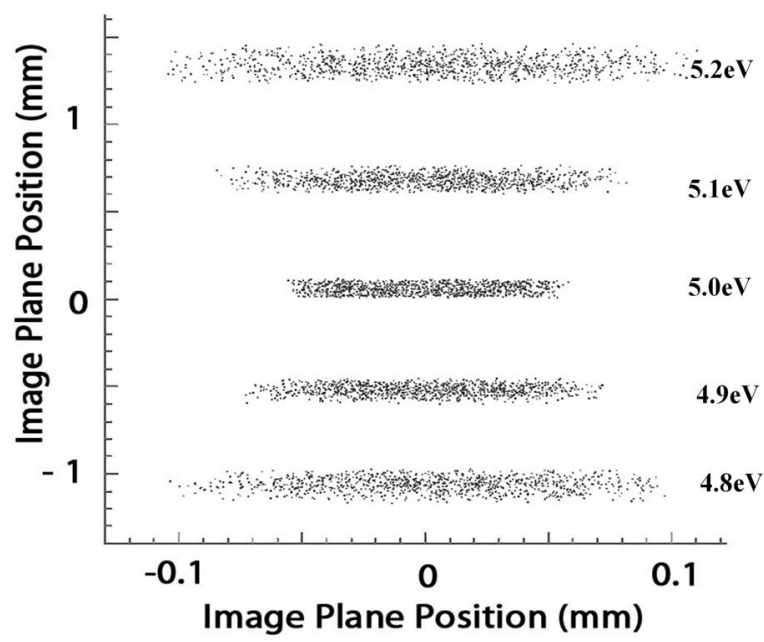

FIG. 3. Raytracing simulation for the optical system showing the image at the exit plane for $4.8-5.2 \mathrm{eV}$ in $0.1 \mathrm{eV}$ increments. entrance slit of the monochromator. To quantify the quality of the focusing, we first measured the source size using a filtered 1:1 achromatic lens system. The result is given in Fig. 4 and shows a somewhat triangular shape, with a size of $135 \mu \mathrm{m}$ (FWHM) wide by $270 \mu \mathrm{m}$ (FWHM) tall image. This shape is due to the fact that the axis of the focusing laser beam that drives the plasma is $60^{\circ}$ off the horizontal light output optical axis.

We then used the double paraboloid refocusing system to relay light to the entrance pinhole and we measured both the focus size and the transmission through the $0.15 \mu \mathrm{m}$ diameter entrance pinhole. The focused size is measured to be $160 \mu \mathrm{m}$ $\times 320 \mu \mathrm{m}$ (FWHM) using a slit scanning beam profiler from Newport. The double mirror system is designed to not need adjustment, through precision manufacture of its mounts, but a critical alignment is that the angle of the light source to the generating axis and the focus of the first paraboloid must be precisely determined. The source is therefore mounted on a

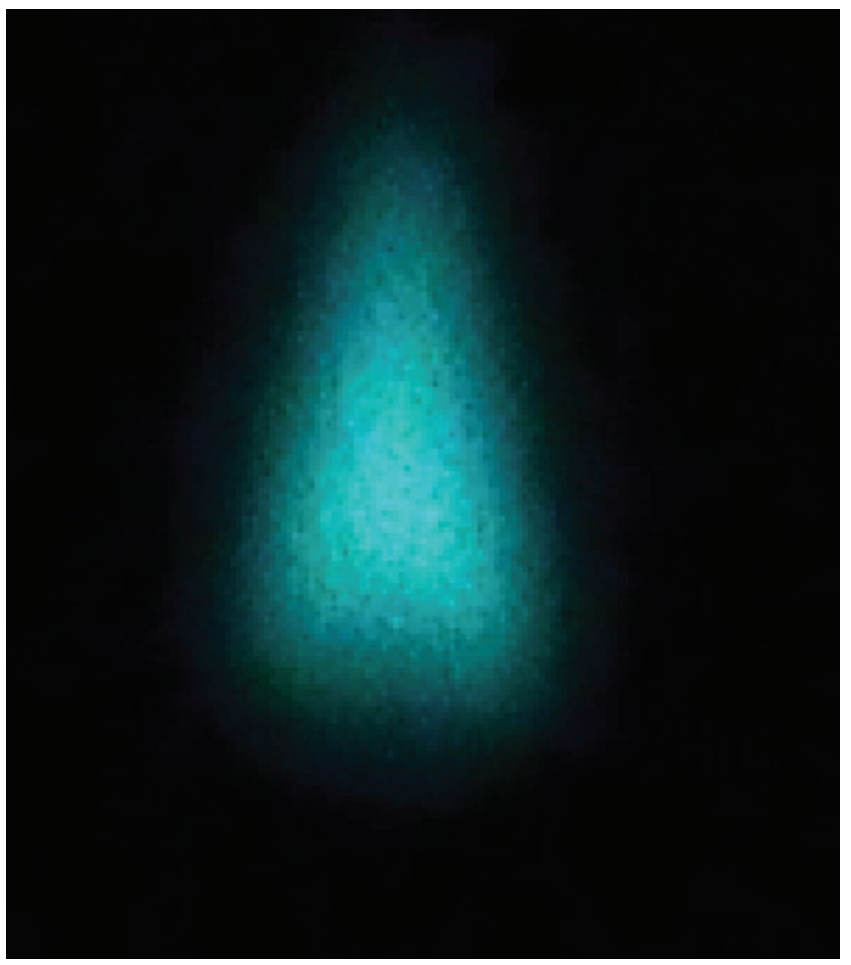

FIG. 4. Emitting area of the plasma light source. The source is measured to be $135 \mu \mathrm{m}$ (FWHM) wide by $270 \mu \mathrm{m}$ (FWHM) tall. 


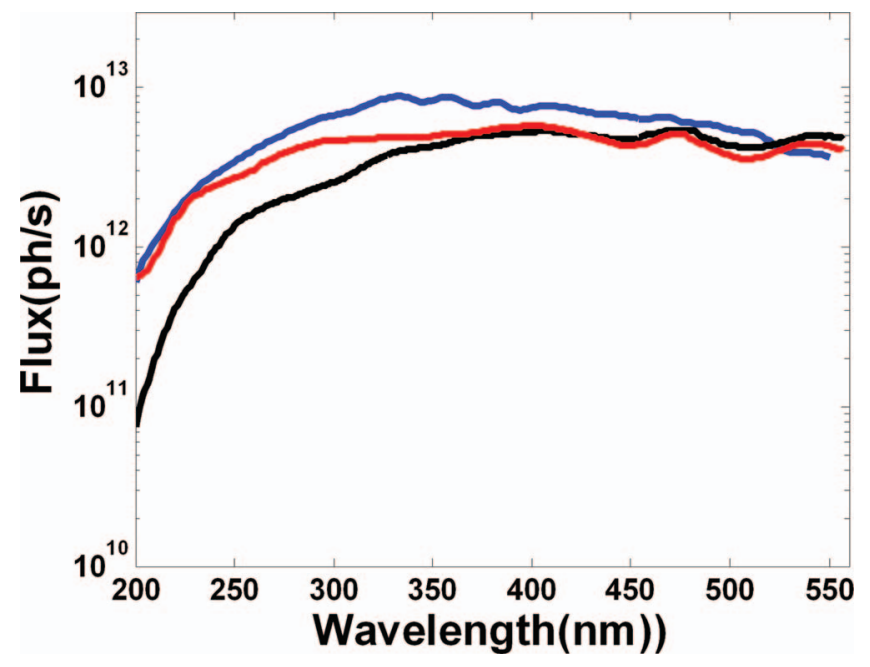

FIG. 5. Measured output flux and its comparison with calculation. Calculated flux (blue), measured flux at the monochromator exit slit (red), and the output coupled through a $1 \mathrm{~m}$ long UV fiber with $100 \mu \mathrm{m}$ core diameter (black) are shown.

small $x-y-z$ stage, and this is translated until the image faithfully represented the source shape. It is found that misalignments of the order of $\pm 0.1 \mathrm{~mm}$ in the transverse plane and $\pm 0.5 \mathrm{~mm}$ in the longitudinal plane are clearly noticeable. After this alignment, the ratio of the integrated intensity be- fore and after the entrance pinhole is measured. The transmission through the $0.15 \mathrm{~mm}$ diameter pinhole is measured to be $20 \%$. The calculated transmission using the measured beam intensity distribution is $22 \%$.

Figure 5 shows the output flux measured with a calibrated Si photodiode and its comparison with calculation results. The red flux curve represents the measurement result by directly coupling the photodiode behind a $100 \mu \mathrm{m}$ pinhole in the monochromator chamber; and the black flux curve was measured through a $100 \mu \mathrm{m}$ core diameter and $1 \mathrm{~m}$ long fiber using the photodiode outside the chamber. The direct output coupling gives higher flux in the UV range than that of fiber output coupling due to the attenuation of the UV transmission of the fiber. The flux is around $6 \times 10^{11}$ photon/s at the wavelength of $200 \mathrm{~nm}$, and increases by roughly an order of magnitude for wavelengths of $300 \mathrm{~nm}$ and greater for direct output coupling.

The expected flux $F_{o}$ can be calculated from the product of the source brightness, the angular collection aperture and all of the transmission and reflectivity coefficients of the optical components, slits, mirrors, and gratings as

$$
\begin{aligned}
F_{o}= & B_{\text {source }} \cdot d \Omega \cdot \lambda_{\text {width }} \cdot T_{\text {window }}^{2} \cdot T_{\text {Entrance_pinhole }} \\
& \cdot R_{O A P}^{4} \cdot E_{\text {grating }} \cdot T_{\text {exit_pinhole }},
\end{aligned}
$$

where $B_{\text {Source }}$ is the light source brightness, $d \Omega$ is the solid angle determined by the off-axis paraboloid mirror aperture
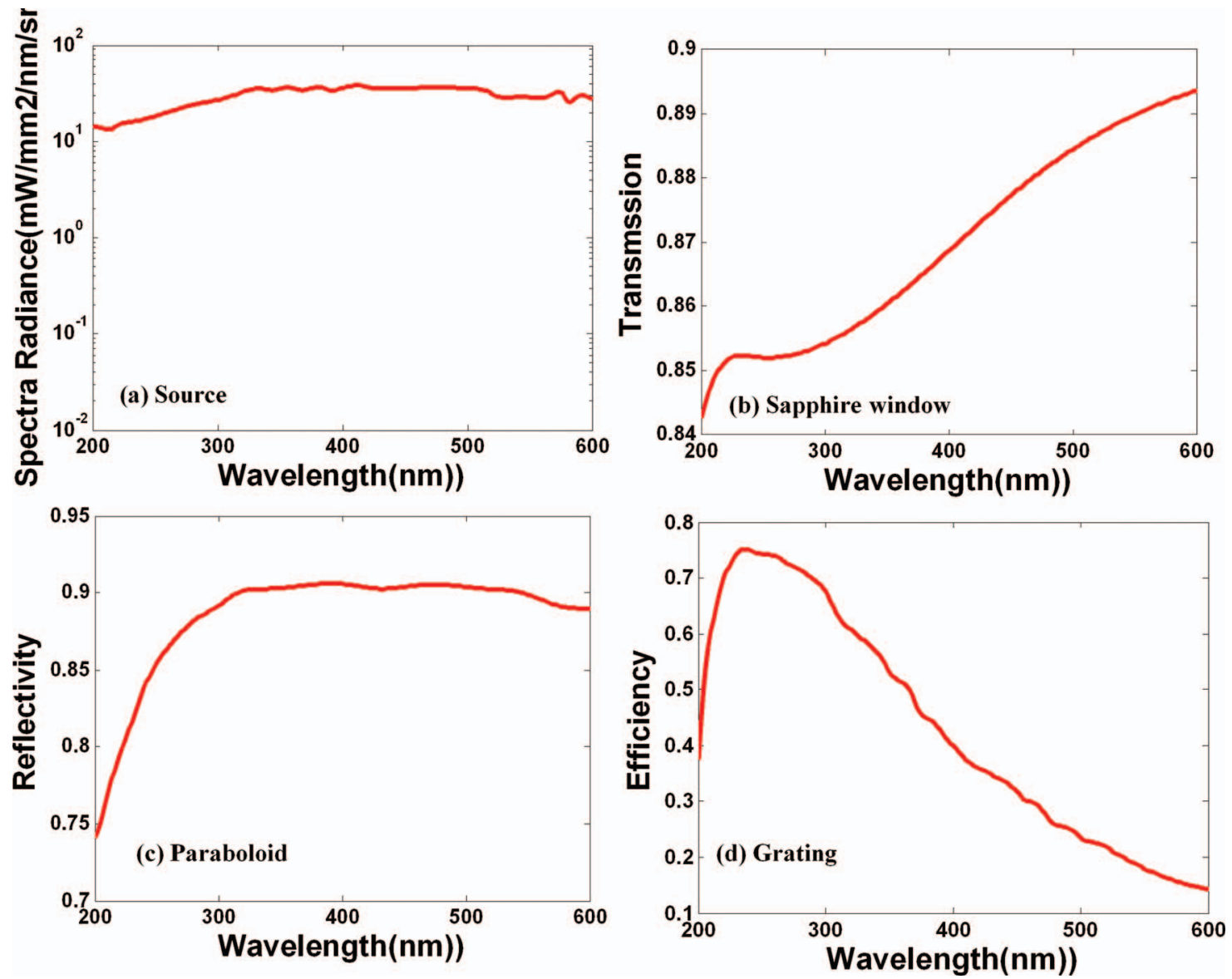

FIG. 6. Spectral response curves of the system components. (a) Source radiance, (b) sapphire window transmission, (c) mirror reflectivity, and (d) grating efficiency. Information on these datasets is given in Ref. 17. 


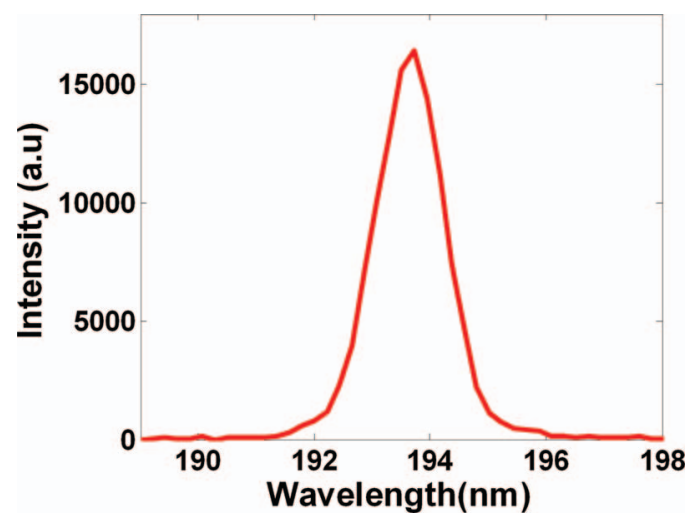

FIG. 7. Measured output spectral centered at $193.7 \mathrm{~nm}$ using Ocean Optics spectrometer.

and source distance, $\lambda_{\text {width }}$ is the spectral bandwidth, $T_{\text {window }}$ is the sapphire window transmission, $T_{\text {entrance_pinhole }}$ is the light transmission through the entrance pinhole, $R_{O A P}$ is the reflectivity of the paraboloid mirror, $E_{\text {grating }}$ is the grating diffraction efficiency, and $T_{\text {exit_pinhole }}$ is the light transmission through the exit pinhole. In our system, there are two sapphire windows and 4 paraboloid mirrors. Figure 6 shows the spectral response curves used in the calculation for (a) the radiance of the plasma light source, (b) transmission of the sapphire window, (c) the reflectivity of the paraboloid mirror, and (d) the diffraction efficiency of the grating. The predicated flux is shown as the blue curve in Figure 5. It can be seen that the measured and calculated flux agrees with each other very well across the whole wavelength range of the monochromator. The flux in the UV for direct output coupling, for wavelengths less than $350 \mathrm{~nm}$, is higher than that in the fiber coupled case, due to fiber absorption losses.

The resolution of the system was measured using a commercial fiber spectrometer (Ocean Optics, USB4000-UV-Vis, $1200 \mathrm{l} / \mathrm{mm}$ grating, $25 \mu \mathrm{m}$ slit), with an intrinsic resolution of $0.75 \mathrm{~nm}$. Figure 7 shows the spectrum of $193.7 \mathrm{~nm}$ light from the system. The measured FWHM in the deep UV is $1.7 \mathrm{~nm}$. The quadratic sum of the entrance and exit slit limited resolution is expected to be $1.5 \mathrm{~nm}$, and combined with the spectrometer resolution we expect a measured width of $1.7 \mathrm{~nm}$, confirming that the monochromator is performing as designed.

Not only should the monochromator and pre-optical system be brightness preserving, but the optical system used for relaying light to an experiment has to be stigmatic and brightness preserving. Toroidal mirrors are often used for the function of relaying light, but for the apertures considered here, geometric aberrations would dominate the focus size. To overcome this disadvantage, we have used an ellipsoidal mirror for this relaying function. In this case we used a $90^{\circ}$ deflecting ellipsoidal mirror with conjugates of 100 and $300 \mathrm{~mm}$. This ellipsoidal mirror has $\mathrm{MgF}_{2}$ over-coated on $\mathrm{Al}$ substrate. For some applications a demagnified image is needed, and for others a magnified and more collimated image is needed. Here we give an example where the system is used in the magnifying/collimating mode to relay the $0.1 \mathrm{~mm}$ exit pinhole source to a final image, at 3:1 magnification. The im-
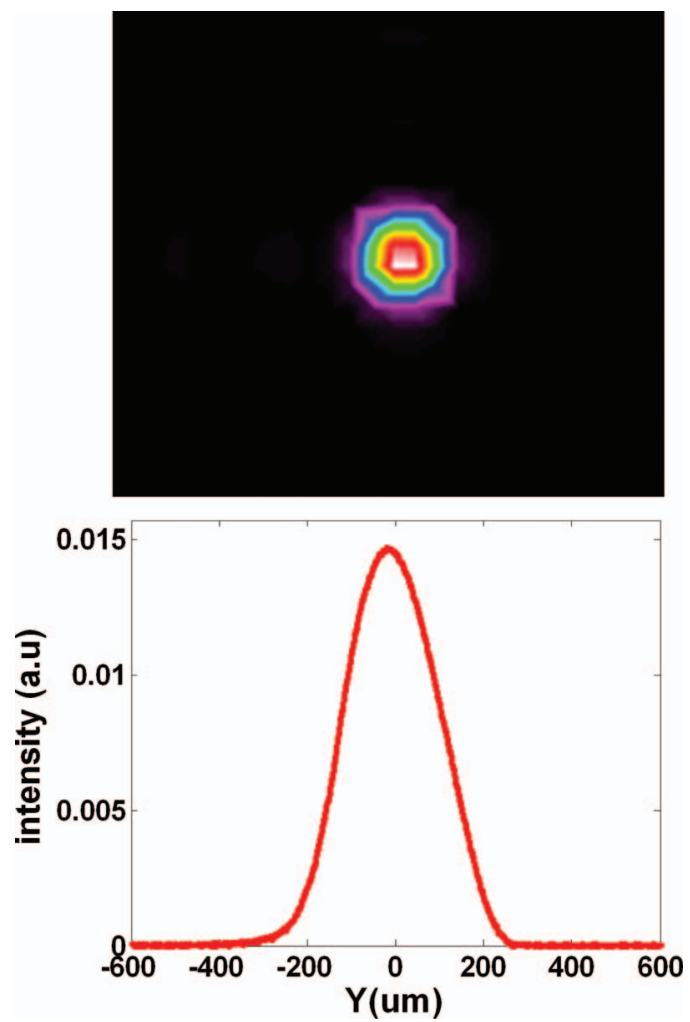

FIG. 8. Measured image using a slit scanning beam profiler (upper panel) and intensity distribution at y direction with $300 \mu \mathrm{m}$ FWHM focus of the 3:1 magnifying ellipsoidal relay mirror (bottom panel).

age was measured using a slit scanning beam profiler and the result is shown in Figure 8. The image has a FWHM of $0.3 \mathrm{~mm}$ and is independent of wavelength. The measurement result shows that the image size is in line with expectations and the chromatic aberration of the ellipsoidal mirror is very small. In order to get this fidelity of imaging, very careful adjustment of the pitch and yaw of the mirror are required.

There are several places where the current system could be further improved. The transmission of the entrance pinhole is lower than expected due to the somewhat larger than expected source size. A lower power version of EQ-1500 source does exhibit smaller source sizes, but total flux is lower. For the $50 \mathrm{~W}$ laser power source used here, the entrance pinhole should be replaced by a slit of $150 \mu \mathrm{m}$ width; also a $150 \mu \mathrm{m}$ core diameter fiber at the exit plane should be used or using a slit to match the entrance slit. This would allow integration of flux over the non-dispersive axis along the full $270 \mu \mathrm{m}$ height of the source. The system was designed for a modest resolution, commensurate with our present applications. Its scale is also commensurate with the size of easily available off-axis paraboloid. However, the system can easily be expanded in scale or the grating line density can be increased to operate at higher resolution, if required.

\section{SUMMARY}

A monochromatic high brightness UV-VIS light system has been successfully developed. The system is brightness preserving and gives $6 \times 10^{11} \mathrm{ph} / \mathrm{s}$ to $4 \times 10^{12} \mathrm{ph} / \mathrm{s}$ in the 
deep UV to visible spectrum range with a spectral resolution of $1.7 \mathrm{~nm}$ delivered into an image size of about $0.1 \mathrm{~mm}$. The system is designed for applications requiring high flux density at the sample and will be useful across a broad field of scientific research from low energy photo-electron emission microscopy (PEEM) and UV photoemission spectroscopy to low energy angle resolved photoelectron spectroscopy (ARPES).

\section{ACKNOWLEDGMENTS}

This work was performed at Lawrence Berkeley National Laboratory under the auspices of the Office of Science, Office of Basic Energy Sciences, of the U.S. Department of Energy under Contract No. DE-AC02-05CH11231. F.J.P. thanks the financial support from the Spanish MINECO (grants MAT2010-18432 and Consolider FUNCOAT CSD2008-0023).

${ }^{1}$ D. H. Dowell, I. Bazarov, B. Dunham, K. Harkay, C. Hernandez-Garcia, R. Legg, H. A. Padmore, T. Rao, J. Smedley, and W. Wan, Nucl. Instrum. Methods Res. A622, 685 (2010).

${ }^{2}$ I. Bazarov et al., J. Appl. Phys. 103, 054901 (2008).

${ }^{3}$ R. Akre et al., Phys. Rev. ST Accel. Beams 11, 030703 (2008).
${ }^{4}$ A. Polyakov, C. Senft, K. F. Thompson, J. Feng, S. Cabrini, P. J. Schuck, H. A. Padmore, S. J. Peppernick, and W. P. Hess, Phys. Rev. Lett. 110, 076802 (2013).

${ }^{5}$ R. K. Li, H. To, G. Andonian, J. Feng, A. Polyakov, C. M. Scoby, K. Thompson, W. Wan, H. A. Padmore, and P. Musumeci, Phys. Rev. Lett. 110, 074801 (2013).

${ }^{6}$ T. Vecchione, I. Ben-Zvi, D. H. Dowell, J. Feng, T. Rao, J. Smedley, W. Wan, and H. Padmore, Appl. Phys. Lett. 99, 034103 (2011).

${ }^{7}$ L. Cultera, I. Bazarov, A. Bartbik, B. Dunham, S. Karkare, R. Merluzzi, and M. Nichols, Appl. Phys. Lett. 99, 152110 (2011).

${ }^{8}$ S. Horne, D. Smith, M. Bessen, M. Partlow, D. Stolyarov, H. Zhu, and W. Holber, Proc. SPIE 7680, 76800L-1 (2010).

${ }^{9}$ T. Namioka, J. Opt. Soc. Am. 49(10), 951-959 (1959).

${ }^{10}$ W. G. Fastie, J. Opt. Soc. Am. 42(9), 641-647 (1952).

${ }^{11}$ M. Czerny and A. F. Turner, Z. Phys. 61(11-12), 792-797 (1930).

${ }^{12}$ D. R. Austin, T. Witting, and I. A. Walmsley, Appl. Opt. 48(19), 3846-3853 (2009).

${ }^{13}$ K.-S. Lee, K. P. Thompson, and J. P. Rolland, Opt. Express 18(22), 2337823384 (2010).

${ }^{14}$ B. Bates, M. MacDowell and A. C. Newton, J. Phys. E: Sci. Instrum. 3, 206-210 (1970).

${ }^{15}$ A. B. Shafer, Appl. Opt. 6(1), 159-160 (1967).

${ }^{16}$ B. Lai and F. Cerrina, Nucl. Instrum. Methods A246(1-3), 337-341 (1986).

${ }^{17}$ (a) Source radiance data were provided by the collaborator H. Zhu from Energetiq Technology, Inc.; (b) sapphire window data were provided by Accu-Glass products, Inc; (c) parabolic mirror used are model50338Al from Newport Corp.; and (d) grating diffraction efficiency was provided by Richardson Gratings. 